\title{
Q-Float in West Ulster English and labeling
}

\author{
Andreas Blümel \\ University of Göttingen \\ ablueme@gwdg.de
}

\begin{abstract}
In this paper I contrast a stranding analysis of Q-float in intermediate $\mathrm{A}^{\prime}$-positions in West Ulster English with the labeling analysis of successive-cyclic $\mathrm{A}^{\prime}$-movement and show that the former represents a problem for the latter. If we want to retain the labeling analysis, alternatives to stranding must come forward, some of which I discuss here.
\end{abstract}

Keywords: Q-Float; Irish; Successive-cyclic Movement; Distributed Deletion.

\section{Introduction}

Sometimes advances in linguistic theory give rise to contradictions or tensions with previously proposed accounts for a phenomenon. In such cases, theoretical parsimony and conceptual elegance can conflict with descriptive adequacy. In principle, such tensions can be resolved in at least two ways: firstly, by discarding or revising the new theory. The former is not desirable or at least begs the question of alternative perspectives. The latter often comes at the cost of enriching an otherwise elegant theory. Secondly, one can discard the hypothesis upon which an analysis is based, or alternatively retain the new theory and show that the effects for the hypothesis are epiphenomenal of other factors. The former requires careful justification and the demonstration that the phenomenon at hand is indeed of a fundamentally different nature. For the latter it is to be hoped that a reanalysis fares at least as good as the old one, descriptively.

The goal of this paper is to revisit evidence advanced in favor of successive-cyclic A'-movement from a dialect of English McCloskey (2000) calls West Ulster English (henceforth WUE), namely quantifier float. I will discuss his Q-stranding analysis of the phenomenon and show that it is incom- 
patible with or at least poses problems for recent considerations of labeling (Chomsky 2013, 2015) and the analysis of successive-cyclic A'-movement it naturally gives rise to (Blümel 2012; Chomsky 2013). I will then sketch analytical alternatives of Q-float in WUE that are consistent with the labelingbased analysis. That said, I will here not be concerned with a discussion and critique of alternatives to a labeling-based analysis of successive-cyclic movement, but refer the reader to Blümel $(2012,2017) .{ }^{1}$

Repeatedly, cross-linguistic or -dialectal variation has helped to support syntactic theory, and the hypothesis of successive-cyclicity is no exception. Moreover, genuine syntactic variation is predominantly captured by reference to parameters, whose form is commonly taken to be functional heads, an immensely fruitful proposal by Hagit Borer. ${ }^{2}$ Q-float in WUE could be considered to simultaneously represent both: it buttresses the idea that long distance $\mathrm{A}^{\prime}$-movement comprises numerous local steps of movement and it doubtlessly minimally contrasts with standard English and is thus a case of cross-dialectal variation. Thus WUE, but not standard English, allows for the following (1b-d).

(1a) What all do you think that he'll say that we should buy?

(1b) What do you think all that he'll say that we should buy?

(1c) What do you think that he'll say all that we should buy?

(1d) What do you think that he'll say that we should buy all?

McCloskey (2000: 62)

$$
\left[\mathrm{CP} 1 \mathrm{what}_{\mathrm{i}} \ldots\left[\mathrm{CP} 2 \mathrm{t}_{\mathrm{i}}(\mathrm{all}) \mathrm{C} 2 \ldots\left[\mathrm{CP} 3 \mathrm{t}_{\mathrm{i}}(\text { all }) \mathrm{C} 3 \ldots \mathrm{t}_{\mathrm{i}}\right]\right]\right]
$$

In examples (1b) and (1c), the quantificational element all, which is associated with the wh-pronoun what, occurs in the respective left peripheries of the embedded clauses. This has been taken to indicate that wh-movement must touch down in these positions such that the long-distance dependency between the wh-phrase and the gap behind the most embedded verb buy is in fact partitioned by - at least - the local clause as schematized in (2).

\footnotetext{
${ }^{1}$ While Cecchetto \& Donati's (2015) approach to successive-cyclic movement might not be confronted with some of the problems mentioned here, their analysis is based on stipulations whose necessity are not clear to me.

${ }^{2}$ But see Obata et al (2015) for an intriguing parameter-free approach.
} 
One question such facts raise is if the variation that we find between standard English and WUE is syntactic, or what the right characterization and analysis of this differences is. I believe that a twofold goal is achieved if it can be shown that an analysis that does not resort to syntactic mechanisms to explain the variation fares better than one that does: we can retain an elegant syntactic theory of successive-cyclic movement, which is cross-linguistically uniform and does not merit complication of analysis on the one hand. On the other hand, we hope to have a descriptively more adequate account of WUE Q-float in wh-questions which takes into account the various intricate properties of the phenomenon (cf. e.g. Rochman 2005 for further phonological aspects of Q-float within standard English alone). In effect, then, we do not need a syntactic parameter for this phenomenon, but might be able to draw on the interfaces between syntax and morphology/phonology alone.

This paper is structured as follows: In Section 2, I will briefly rehash some of the pieces of evidence for successive cyclic $\mathrm{A}^{\prime}$-movement and the general analyses that have been proposed. Section 3 lays out the recent labeling-based alternative in Blümel (2012) and Chomsky (2013), and demonstrates that McCloskey's approach to capture Q-float in WUE is at variance with this solution. Section 4 discusses possible alternative analyses of Q-float in WUE that could salvage the labeling analysis. In Section 5 phonological factors are briefly laid out, which doubtlessly play a role in the phenomenon. Section 6 concludes the paper.

\section{Successive cyclic $A^{\prime}-$ Movement}

As noted above, there is a principled possibility to dismiss the successivecyclicity hypothesis, in which case the problem posed by Q-float in WUE must be recast altogether. However, I believe that there are good reasons to retain the idea, despite (rare) claims to the contrary (cf. den Dikken 2009 for the rejection of the idea of successive cyclic movement via SPEC-CP).

Let me briefly rehash some of the pieces of evidence for the successive cyclicity hypothesis. The hypothesis says, in brief, that long-distance dependencies involve a derivation like in (3), not (4), where movement targets the matrix SPEC-CP directly from the launching site, the complement position of the embedded verb.

$$
\text { [CP what } \left.\left.t_{i} \text { do you think [CP } t_{i} \text { that Mary did } t_{i}\right]\right]
$$


(4) $\quad\left[\right.$ CP what $t_{i}$ do you think that Mary did $\left.t_{i}\right]$

There is a widespread challenge and intuition, namely that intermediate movement steps are not feature-driven or indirectly feature driven at best (cf. Bošković 2007). For example, semantic evidence comes from the possibility of having intermediate binding options (Barss 1986), as in (5-6).

(5) Who said that $\mathrm{John}_{\mathrm{i}}$ thinks that Bill $\mathrm{j}_{\mathrm{j}}$ bought pictures of himself $*_{*_{i} j}$ ?

(6) [Which pictures of himself $\left.f_{i j}\right]$ does John $_{i}$ think that Bill $l_{j}$ bought?

English

(5) shows a violation of Principle A of the Binding Theory in that the reflexive pronoun inside the complex wh-phrase is outside the binding domain of the antecedent and thus structurally too remote to be bound by the subject of the matrix clause. $\mathrm{A}^{\prime}$-moving the complex NP into the matrix clause (cf. 6) remedies this situation and creates a new binding option. A plausible interpretation is thus that the complex NP undergoes an intermediate landing in the embedded SPEC-CP position where Principle A applies locally.

Word order facts from Belfast English likewise support the conclusion (Henry 1995), as in (7-8).

(7) Who did John hope [did we see]?

(8) What did Mary claim [did they steal]?

The standard reasoning is that if subject-auxiliary inversion is contingent on local wh-movement, these data too support the successive cyclicity hypothesis in that inversion is triggered by local $\mathrm{A}^{\prime}$-movement of what and who respectively into the embedded SPEC-CP position. A'-movement is subsequently completed by movement from the intermediate SPEC-CP to matrix SPEC-CP.

Fairly visible or audible evidence comes from repeated discontinuous pronunciation of the $\mathrm{A}^{\prime}$-moved element in the very position that we would expect - embedded SPEC-CP - if the successive-cyclicity hypothesis is correct: wh-copying in German and other Germanic languages, such as Afrikaans (cf. du Plessis 1977; Felser 2004), is often cited to support the hypothesis, as in (9). 
(9) Wen denkst Du wen Maria gesehen hat?
who think you who Mary seen
'Who do you think Mary has seen?'

Suggestive morphological evidence also comes from complementizer alternations in Irish (McCloskey 2001). Here the default form of the complementizer is go (glossed as GO) as in the embedded declarative clause in (10).

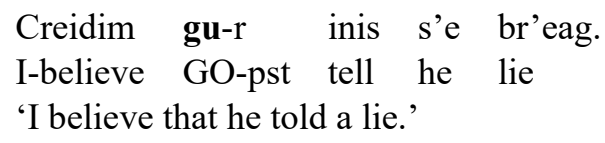

Example (11) represents a sentence with a local A'-dependency, namely a relative clause. The $\mathrm{A}^{\prime}$-movement dependency is established between a silent relative operator and the direct object gap position, following the subject-DP na siogai 'the fairies'. In this case, the complementizer must be realized as $a$ (boldfaced, and glossed as aL), as (11) shows.

$$
\begin{aligned}
& \text { [an ghirseach] a ghoid na } \text { siogai } \\
& \text { the girl aL stole the fairies } \\
& \text { 'the girl that the fairies stole away' }
\end{aligned}
$$

Strikingly, this form also shows up in intermediate C positions (12).

$$
\begin{aligned}
& \text { rud a gheall tu a dheanfa } \\
& \text { thing aL promised you aL do } \\
& \text { 'something that you promised that you would do' }
\end{aligned}
$$

If this complementizer alternation is contingent on the presence of a local $\mathrm{A}^{\prime}$ dependency, this can be taken as an indication that the relative operator has undergone an initial local movement step into the subordinate SPEC-CPposition, before moving on to the matrix $\mathrm{CP}$.

There are various approaches to capture this distributonal pattern of successive cyclic A'-movement, such as conditions on the formation of chains (Takahashi 1994; Boeckx 2003), or the idea that movement is driven by a requirement on the moving element plus phases, a promise that is ultimately redeemed in the final landing position. As noted, I will not go into a critique of these approaches but merely note that a simpler one is to be had. While the 
cross-linguistic evidence in favor of successive-cyclic A'-movement strikes me as strong, specific grammatical analyses of the actual phenomena associated with this syntactic dependency can be complex. In the following section I will describe what strikes me as the simplemost syntactic approach to successive-cyclic A'-movement and show that one standard approach to the evidence for intermediate landing sides is at odds with that syntactic approach.

\section{Stranding and the labeling analysis of successive cyclic movement}

In this section, I would like to briefly characterize the framework developed by Chomsky $(2013,2015)$, describe the analysis of successive-cyclic $\mathrm{A}^{\prime}$ movement and then show a problem related to McCloskey's stranding analysis of Q-float.

The core assumption about the structure building operation Merge in Chomsky $(2013,2015)$ is that the procedure is as simple as it possible, i.e. it does no more than create unordered sets of the arguments it applies to and, crucially, no mechanism of projection is involved. This is shown in (13).

$$
\operatorname{Merge}(X, Y)=\{X, Y\}
$$

However, the notion of "most prominent element" in a given unit does not go out of the window entirely. By assumption, labels are needed for interpretation and selectional purposes, i.e. while endocentricity is dispensable with respect to Narrow Syntax, the Conceptual Intentional systems require an answer to the question: "What kind of unit is it?" So how can we account for this, given the minimal syntax exemplified in (13)? According to Chomsky (2013), a Third Factor principle Minimal Search operates to achieve this: At a given phase level, a labeling algorithm, which abides by Minimal Search, detects the most prominent element - the label - in a given set. This is shown by underlining the relevant element in (14). If Merge applies to a head and a phrase, the following result obtains from the application of the labeling algorithm, shown in (14).

$$
\{\mathrm{X}, \mathrm{YP}\} \rightarrow\{\underline{\mathrm{X}}, \mathrm{YP}\}=\mathrm{XP}
$$

This is the trivial case in that even though Merge per se is strictly symmetric, the structural complexity of the members is not: The simplex element $\mathrm{X}$ is 
immediately detectable while $\mathrm{YP}$ - itself a set $\{\mathrm{Y} \ldots\}$ - requires deeper search. After application of the labeling algorithm, the set above behaves as $\mathrm{XP}$.

Of course, Merge can furthermore apply to two heads or two phrases respectively, each of which involves elements of equal complexity. The following condition applies in the first case:

(15a) $\{\mathrm{X}, \mathrm{Y}\} \rightarrow\{\underline{\mathrm{X}}, \mathrm{Y}\}$, iff $\mathrm{Y}$ is a categoryless root and $\mathrm{X}$ a categorizer $(n$, $v, a)^{3}$

(15b) $\quad\{\underline{X}, Y\}=X P$

Merger of two phrases delivers a set $\{\mathrm{XP}, \mathrm{YP}\}$ in which no element is more prominent than the other: Is XP a specifier of Y, or is YP a specifier of X? No answer can be given in this framework, because projection, and consequently the very notion specifier, is unavailable. For this case, at least two solutions have been proposed to solve the ensuing labeling problem, but I will here describe only one that I will dub symmetry-breaking movement, ${ }^{4}$ while I leave aside labeling under feature sharing.

The idea is that movement creates a discontinuous syntactic object - a movement chain - of which lower members are invisible to syntactic operations (broadly speaking). If so, the labeling algorithm is blind to lower copies/occurrences of a movement chain. ${ }^{5}$ Consider, for example, a VP-internal subject, which is rendered as an NP within an unlabeled set $\{\mathrm{NP}, \nu \mathrm{P}\}$. If NP raises to a higher position, the lower copy of NP is invisible in $\{<\mathrm{NP}\rangle, v \mathrm{P}\}$, while the head of the movement chain remains visible to syntactic operations. Consequently, this set is labeled by $v \mathrm{P}^{6}$ In this sense, movement appears to "collapse" structure, at least as far as operations like the search for a

\footnotetext{
${ }^{3}$ Cf. also Alec Marantz' recent work, as well as Irwin (2012), a.o., for the idea that lexical roots do not project.

${ }^{4}$ Cf. Moro (2000).

${ }^{5}$ Cf. Chomsky (2000: 115).

${ }^{6}$ Given this line of reasoning, "deep search" appears to be required in the sense that at least one complex object must be inspected to retrieve the information of the head of $v \mathrm{P}$, namely $v$. As far as I can see, this is consistent with the ideas in Chomsky (2013/2015) in that he proposes something similar for the "shared feature" solution of labeling $\{\mathrm{XP}, \mathrm{YP}\}$, where both members must be inspected in parallel as it were. Notice also that more must be said about the issue that non-topmost copies in a movement chain are invisible to the search for a label.
} 
label are concerned. A question that arises is what happens in the target of movement. Suppose, for example, that a wh-NP who raises to the sister position of a non-interrogative $\mathrm{CP}$ to yield $\alpha=\{\mathrm{NP}, \mathrm{CP}\}$, as in (16).

$$
\text { John say }\left[{ }_{\alpha} \mathrm{NP}=\text { who }\left[{ }_{\mathrm{CP}} \text { that Mary met }<\text { who }>\right]\right]
$$

As can be seen, Minimal Search for a label cannot succeed in that both members in $\alpha$ are of equal complexity. The idea then is that $\alpha$ 's labeling problem is solved by again moving who higher up into the matrix clause, i.e. by reiterating symmetry breaking movement (cf. Blümel 2012; Chomsky 2013). This, of course, is the exact pattern we see in successive-cyclic movement in that the phenomenon is unbounded in principle, just as the mechanism described. I refer the reader to the works cited for solutions to the halting problem, i.e. possible ways to escape the loop of symmetry creating by symmetry breaking.

Let us now address the challenge this analysis raises for Q-float in WUE. McCloskey (2000) suggests what I will refer to as a stranding account. This is shown with a complex DP in (17), which I dub DP1 for exposition. The quantifier heads DP1 and a separate DP2 occupies its specifier - is the category of the wh-pronoun. Q-float is restricted to pronominal wh-phrases, i.e. full wh-phrases do not permit Q-float. ${ }^{7}$

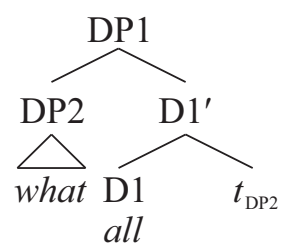

Q-float in intermediate positions then involves movement of DP1 to the embedded SPEC-CP and subsequent subextraction of DP2 towards higher CPSPEC-positions as shown in $(18 \mathrm{~b})$. Stranding D1=all in this intermediate

\footnotetext{
${ }^{7}$ McCloskey (2000) does not provide ungrammatical cases with complex wh-phrases, but the restriction is implicit in his remarks.
} 
SPEC-CP position yields the surface distributional profile of WUE Q-float (cf. McCloskey 2000: 62), as in (18).

(18a) What do you think all that we should buy?

(18b)

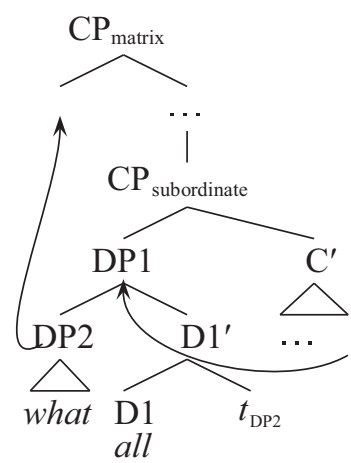

Now a labeling-based account of successive-cyclic A'-movement raises the question how the embedded $\mathrm{CP}$ gets its label in the first place. Remember that label-detection applies at a respective phase level and the status of what used to be $\mathrm{X}^{\circ}$, XP and $\mathrm{X}^{\prime}$ levels is determined dynamically and derivationally: $\alpha$ in (19) bears no label unless DP1 in toto vacates $\{\mathrm{DP} 1, \mathrm{CP}\}$.

(19)

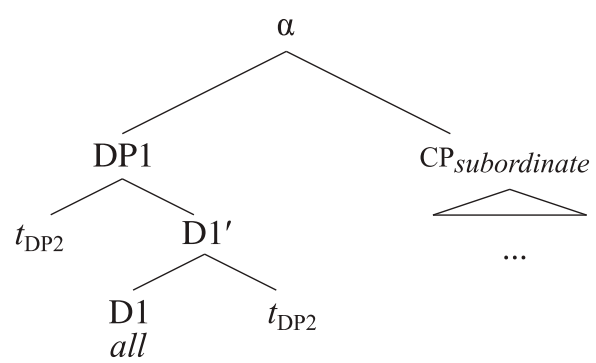

The D1-head all - but not the subextracted portion - is the head of the movement chain and is thus labeling-visible: the labeling algorithm cannot assign the subordinate structure $\{\mathrm{DP} 1, \mathrm{CP}\}$ the category $\mathrm{CP}$, because the syntactical- 
ly stranded DP1 is an equally available candidate. ${ }^{8}$ The possibility is conceivable that stranded all might be a head instead of a phrase due to the evacuation by DP2, i.e. the resulting structure is $\{\mathrm{D} 1, \mathrm{CP}\}$ after subextraction. That too raises a question: is this structure labeled by D1? This is an expected result, but arguably an undesireable one, given that the resulting category will be selected by the matrix verb think. The problem is exacerbated once we think of comparable constructions like exactly-float (cf. Urban 1999), like in What do you think exactly that Mary has read? If exactly is (a) a head and (b) stranded in an intermediate sister-of-CP-position, the stranded adverb will determine the category in the set $\{\mathrm{Adv}=$ exactly, $\mathrm{CP}\}=\mathrm{AdvP}$. A matrix verb will then select this AdvP - which we know is not independently possible.

Of course, there is a way around this problem by saying that both quantifiers in simplex wh-phrases and adverbs like exactly are adjuncts. One could then imagine a late Merger (cf. i.a. Fox 2002) account of these adverbs where these adjuncts merge late with the (unpronounced) copies of the wh-phrases in the intermediate positions. However, I still feel that this is not satisfying, mainly for empirical reasons: Wiland (2010) observes that floating of nouns in intermediate positions of long distance Left Branch Extraction is possible in Polish. Such cases leave little room for this kind of explanation (late Merger). Provided these phenomena deserve a uniform analysis, we have to look for alternatives.

Summing up this section, given a labeling-analysis, a stranding account makes wrong predictions: either the structure is not interpretable by the Conceptual Intentional systems or Q-float should not be possible, both of which are wrong. In this section I have identified a problem of a (naïve view of the) stranding analysis of Q-float in WUE for the labeling analysis of successivecyclic $\mathrm{A}^{\prime}$-movement. I will now turn to conceivable solutions.

\section{Conceivable solutions}

In this section I will sketch and briefly discuss possible alternative analyses of WUE Q-float which are consistent with the labeling analysis of successive-cyclic $\mathrm{A}^{\prime}$-movement.

\footnotetext{
${ }^{8}$ That technical problem aside, the analysis raises questions about freezing: To what extent is subextraction from a displaced XP licit (cf. Corver 2007)? The issue is much debated and strong conclusions are inexpedient (cf. Boeckx 2008: 80ff).
} 


\subsection{Floating quantifiers as adverbs}

The literature on Q-float is roughly divided into two camps: One camp argues that the phenomenon comes about by stranding, as detailed above and repeated in (20a). The other group of researchers argues that floating quantifiers are best analyzed as adverbially used phrases, which adjoin to the VP as sketched in (20b). I here gloss over differences of implementation within a given approach.

(20a) [The students $]_{i}$ have [vP [DP all $\left.t_{i}\right]$ seen the movie $\left.]\right]$

(20b) [The students $]_{i}$ have [vp all [vp $t_{i}$ seen the movie]]

Importantly, if the adverbial approach turns out to be correct for Q-float in WUE, it might have no bearing on the successive-cyclicity hypothesis at all. I know of no worked-out analysis to that effect (cf. Bobaljik 2003: 3.3. for a remark).

The analyses above make distinct predictions and each account for different properties of Q-float cross-linguistically. Fitzpatrick (2006) pursues a mixed approach and argues at length that both strategies must be available cross-linguistically but sometimes also language-internally (like e.g. Russian). He suggests that certain correlations exist in the ways that languages employ Q-float, which are listed in Table 1 (from Fitzpatrick 2006: 37).

Table 1.

\begin{tabular}{lll}
\hline & $\begin{array}{l}\text { Adnominal floated } \\
\text { quantifier }\end{array}$ & $\begin{array}{l}\text { Adverbial floated } \\
\text { quantifier }\end{array}$ \\
\hline Distribution & NP/DP positions & adverbial \\
Movement correlation & A'-movement & A-movement \\
Origin & Stranded & Not stranded \\
Semantics & Non-exhaustive & Exhaustive \\
\hline
\end{tabular}

Other language-particular properties add to the picture, such as whether or not the quantifier agrees with its associate in Phi-features and case. Insofar as these correlations hold, they give rise to the straightforward expectation that Q-float in WUE belongs to the left column and should be analyzed as envisaged by McCloskey. 
With respect to the semantics of Q-float, Fitzpatrick shows that the correlation indeed holds. First of all, he cites McCloskey in personal communication (Fitzpatrick 2006: 156), saying that Q-float does not differ from continuous wh-phrases plus quantifiers with respect to its meaning. It has been suggested that the addition of the quantifier adds to the meaning of the question that the possible answers be listed exhaustively, a requirement that is absent when all is missing (McCloskey 2000: 58). However, Fitzpatrick (2006: 155-162) argues that the facts are more nuanced. Based on examples like (21) and (22) he argues that the semantic contribution of all is in fact weaker: the quantifier functions as a semantic pluralizer (cf. also Reis 1992 with respect to German invariant alles).

(21a) John told me where all to buy the New York Times.

(21b) \#No he didn't. He didn't tell you about Out of Town News.

(22a) Where all did you get that outfit that you're wearing?

(22b) \#Where all did you get those shoes that you're wearing?

The response to (21a) given in (21b) is strange. But it is predicted to be good if an exhaustive list of places where one can buy the New York Times were required by the use of all, "however improbable the situation" (Fitzpatrick 2006: 161). Moreover, given that, usually, shoes are not bought individually but in pairs, it is strange to ask for the plurality of places where the addressee got his or her shoes - however, this is what all semantically contributes in (22). Neat as the approach is, it crucially hinges on the correctness of the correlation established by Fitzpatrick. Additional confirming or disconfirming evidence is required to strengthen or weaken that idea, especially with respect to Q-float in WUE.

Notice that distributional facts could challenge an adverbial approach to Q-float in WUE, possibly plus extraposition of the embedded clause, an idea Bobaljik (2003: 3.3.) adumbrates in passing without giving specific examples where the operation should apply: quantifiers can occur between indirect objects and subordinate object clauses (McCloskey 2000: 63), as in (23).

?What ${ }_{i}$ did he say to him all (that) he wanted to buy $\mathrm{t}_{\mathrm{i}}$ ?

We might assume that the prepositional object is in the complement position of the root or V-position of say and the clausal argument in its SPEC, as in (24). 


$$
[v[\mathrm{CP}[\mathrm{V} / \mathrm{R}=\text { say } \mathrm{PP}=\text { to him }]]]
$$

Now, we might say that all adjoins rightward at the VP- or $v$ P level. However, this begs the question how the subordinate clause arrives at its surface position: finite embedded clauses with null-C are known not to undergo extraposition and yet are possible, following all. All of McCloskey's examples involve $\mathrm{CPs}$ optionally with $\mathrm{C}=$ that or $\mathrm{C}=\varnothing$. If extraposition of the $\mathrm{CP}$ is somehow involved in the analysis, this consistent behavior surely needs addressing. Alternatives are conceivable, of course.

To conclude, I have briefly gone into the possibility of analyzing WUE Q-float terms of adverbials which adjoin to the VP. The issue is open.

\subsection{Distributed deletion}

A labeling-account of successive cyclic $\mathrm{A}^{\prime}$-movement can easily be retained if WUE Q-float is analyzed as Distributed Deletion (DD, Fanselow \& Cavar 2002). DD crucially involves copying as the device for movement. Abstractly put, DD opens the possibility that the pronunciation of members of a movement chain can be scattered, instead of exclusively pronouncing the topmost member, which is the default. This means that under narrowly defined circumstances, parts of a lower copy can be pronounced while they are deleted in the higher copy. This is schematized in (25) where strikeout indicates phonological deletion.

$$
[\mathrm{XP} Y \mathrm{X} X] \ldots[\mathrm{XP} Y \mathrm{P} X]
$$

As can be seen, deletion of only YP in the upper copy of XP goes hand in hand with complementary deletion of $\mathrm{X}$ in the lower copy of XP, and pronunciation of YP within XP. As said, DD crucially requires that movement involves copying. Let me make a remark on this issue. In my view, there are very good independent conceptual reasons to assume copies more generally: External Merge (EM) involves copying. Whenever EM applies to an item from the lexicon, a copy is created which is used in the derivation. Not assuming this much would be absurd. ${ }^{9}$ However, the same conclusion is commonly not drawn for Internal Merge (IM), where alternative conceptions

\footnotetext{
${ }^{9}$ Matters differ if one wants to resort to notions like Numeration, which I abstract away from here, cf. Epstein et al. (2014) on the dispensability of the concept.
} 
have been proposed in the literature, such as multi-dominance. Alternatively, if IM does not involve copies, an artificial asymmetry between EM and IM effectively is introduced.

Pursuing a DD-approach to Q-float in WUE would amount to saying that its syntax is the same as that of standard English: Syntactically and crossdialectally, the complex DP is compelled to move in full as required by labeling. However, phonologically and dialect-specifically, pronunciation of the relevant material superficially yields the possibility of a discontinuous pattern in WUE, as in (26).

$$
\text { [CP2 [DP what all }][\mathrm{C} 2 \ldots[\mathrm{CP}[\mathrm{DP} \text { what all }]][\mathrm{C} 1 \ldots[\mathrm{DP} \text { what all }]]]
$$

By contrast, standard English disallows phonological deletion of material in the upper copy of wh-phases (we know that it might allow it for non-whphrases), i.e. it does not employ DD.

DD doubtlessly requires constraints on its application. Fanselow \& Cavar (2002) propose a pragmatic constraint that yields DD whenever conflicting grammatical requirements force pronunciation in different positions, shown in (27).

\section{Condition on DD}

$\mathrm{XP}$ bears a feature $\mathrm{fl}$ that requires that XP be overtly realized in position $\mathrm{A}$, and an additional feature $\mathrm{f} 2$ that forces $\mathrm{XP}$ into position $\mathrm{B}$. Then XP is split up.

One question to ask at this point is which features play a role in WUE. As a starting point, it appears plausible to say that pronunciation of the topmost copy is always the default option, across dialects and languages. We would thus like to understand when the default is suspended. There appear to be phonological conditions on Q-float, which I will go into in the next section. However, as for morphosyntactic features which might condition DD, no candidate has come forth. ${ }^{11}$ Let me emphasize that at least in principle, DD

\footnotetext{
${ }^{10}$ The ideas just stated require additional considerations for cases in which EM does not target the lexicon, but a separate workspace. I refer the reader to Chomsky et al. (2017) for recent elaborations and a possible solution.

${ }^{11}$ In her paper on phonological conditions on Q-float, Rochman (2005: 328) suggests that a [+contrast] feature might play a role in the distribution of quantifiers. While this might be the
} 
is a possible solution to reconcile a labeling analysis of successive-cyclic movement and Q-float. It strikes me as suggestive to pursue an approach that says that copies of constituents may receive a "non-constituent" pronunciation just as it has been suggested that cases of ellipsis might be cases of nonconstituent pronunciation (Ott \& Struckmeier 2018 and references therein). That said, let me make a remark on the role prosody plays in Q-float.

\section{A remark on the role of prosody}

McCloskey (2000: 60/61) states that "wh-quantifier float examples [...] are optimal when all can be incorporated into a preceding head, preferably a verb". The relevant literature leaves little doubt that prosody plays an important role in the distribution of Q-float in WUE and the complete picture of Q-float is much more involved than I have shown up to this point. McCloskey (2000b) makes more specific suggestions and proposes that "the right boundary of an XP [is crucial] in the mapping between syntactic representations and prosodic representations." His approach is summarized here:

- "[T]he rightmost element of a Phonological Phrase in English is prosodically prominent." The phonological structure of a wh-question with a floated quantifier is given below in (28).

- all is prosodically weak, at least, when combined with a wh-pronoun. ${ }^{12}$

- Thus there is a conflict between the Phonological Phrase requirement and the intrinsic weakness of the quantifier. This contradicting requirements are resolved by incorporating the quantifier into the preceding verb such that the two form a phonological word. By itself, the quantifier cannot bear out both its intrinsic prosodic weakness and the condition that the rightmost element of a Phonological Phrase is prosodically prominent.

case for standard English Q-float in non-wh-contexts, WUE represents an inadequate area of application as these wh-questions do not involve contrastive focus.

${ }^{12} \mathrm{He}$ (McCloskey 2000b: 3 ) contrasts this with personal pronouns where all can clearly be prosodically prominent: $y^{\prime}$ all. 
- Following Selkirk (1995: 565), who holds that "[t]he most prominent syllable of an edge constituent is more prominent that that of a constituent not located at an edge," he suggests that the phonological word get all plays the role of such an edge constituent.

$$
\begin{array}{llll}
\text { What } & \text { did } & \text { he get all for Christmas? }
\end{array}
$$

This way, McCloskey (2000: 66) is able to account for fairly intricate patterns and phonological conditions seem to me to surely play a key role in them. The paradigm in (29-31) exemplifies this.

(29a) ?Who did you talk to all?

(29b) ?Who was he laughing at all?

(30) ?*Who were you setting beside all?

(31a) ?Who did you give tea to all?

(31b) *Where did you move the books to all?

The contrast between (29) on the one hand and (30) on the other exemplifies that Q-float crucially depends on the phonological simplicity of the preposition: float behind bisyllabic beside is impossible, while float after monosyllabic to and at is fine. Likewise, float is possible after a direct object that is phonologically simplex, like tea (31a). But a complex one like the books renders Q-float ungrammatical (31b). In each case we can say that Q-float can happen as long as incorporation of the quantifier is possible, i.e. as long as the phonological condition above are met. ${ }^{13}$ These conditions appear to be necessary when it comes to accounting for Q-float in base positions, but they appear to be violated in intermediate SPEC-CP positions. This, in turn, indicates that syntactic factors are necessary; phonological ones must be added in base positions and must not be added in intermediate positions.

\footnotetext{
${ }^{13}$ Rochman (2010: 5) identifies further phonological conditions: the item in question must be monosyllabic, onsetless (words without onset tend to resyllabify so that they gain an onset) and function words.
} 


\section{Conclusion and questions}

This paper has discussed a stranding account of Q-float in West Ulster English in regard to the labeling analysis of successive-cyclic movement and shown that the two are not straightforwardly compatible. Alternative analyses have been hinted at and discussed; in particular a Distributed Deletion analysis in addition to phonological factors appears to me a promising avenue to take. A full-fledged analysis awaits which takes into account more, and hopefully decisive, arguments.

\section{References}

Barss, A. 1986. Chains and anaphoric dependence: On reconstruction and its implications. (PhD dissertation, MIT, Cambridge.)

Blümel, A. 2012. "Successive cyclic movement as recursive symmetry-breaking”. In: Arnett, N. and R. Bennett (eds.), Proceedings of the 30th West Coast Conference on Formal Linguistics. 87-97.

Blümel, A. 2017. Symmetry, shared labels and movement in syntax. Berlin: Mouton de Gruyter.

Bobaljik, J.D. 2003. "Floating quantifiers: handle with care". In: Cheng, L. and R. Sybesma (eds.), The second Glot International state-of-the-article book: The latest in linguistics. Berlin: Mouton de Gruyter. 107-148.

Boeckx, C. 2003. Islands and chains: Resumption as stranding. Amsterdam: John Benjamins.

Boeckx, C. 2008. Understanding minimalist syntax: Lessons from locality in longdistance dependencies. Oxford: Blackwell.

Bošković, Ž. 2007. "On the locality and motivation of Move and Agree: An even more minimal theory". Linguistic Inquiry 38(4). 589-644.

Chomsky, N. 2000. "Minimalist inquiries: The framework". In: Martin, J.U.R. and D. Michaels (eds.), Step by step: Essays in syntax in honor of Howard Lasnik. Cambridge, MA: MIT Press. 89-155.

Chomsky, N. 2013. "Problems of projection". Lingua 130. 33-49.

Chomsky, N. 2015. "Problems of projection, extensions". In: Di Domenico, E. and S. Matteini (eds.), Structures, strategies and beyond: Studies in honour of Adriana Belletti. Linguistik Aktuell/Linguistics Today.

Chomsky, N., Á.J. Gallego and D. Ott. 2017. "Generative grammar and the faculty of language: Insights, questions, and challenges". Revised version to appear in Catalan Journal of Linguistics.

Corver, N. 2007. "Subextraction”. In: Everaert, M. and H. van Riemskdijk (eds.), The Blackwell syntax compantion. Oxford: Blackwell.

den Dikken, M. 2009. On the nature and distribution of successive cyclicity. Paper presented at NELS 40, MIT, November 2009. 
Du Plessis, H. 1977. Wh-Movement in Afrikaans. Linguistic Inquiry 8. 723-726.

Epstein, S.D., T.D. Seely and H. Kitahara. 2014. Labeling by minimal search: Implications for successive cyclic A-movement and the conception of the postulate 'phase'. Linguistic Inquiry 45(3). 463-481.

Fanselow, G. and D. Çavar. 2002. Distributed deletion. In: Alexiadou, A. (ed.), Theoretical approaches to universals. Amsterdam: Benjamins. 65-107.

Felser, C. 2004. Wh-copying, phases, and successive cyclicity. Lingua 114. 543-574.

Fitzpatrick, J.M. 2006. The syntactic and semantic roots of floating quantification. (PhD dissertation, MIT, Cambridge, MA.)

Fox, D. 2002. Antecedent-contained deletion and the copy theory of Movement. Linguistic Inquiry 33(1). 63-96.

Henry, A. 1995. Belfast English and Standard English: Dialect variation and parameter setting. OUP: Oxford.

Irwin, P. 2012. Unaccusativity at the interfaces. (PhD dissertation, NYU.)

McCloskey, J. 2000a. Quantifier float and Wh-movement in an Irish English. Linguistic Inquiry 31. 57-84.

McCloskey, J. 2000b. The prosody of quantifier stranding under WH-movement in West Ulster English. (Ms., University of California, Santa Cruz.)

McCloskey, J. 2001. The morphosyntax of Wh-extraction in Irish. Journal of Linguistics 37. 67-100.

Moro, A. 2000. Dynamic antisymmetry. Cambridge, MA: MIT Press.

Obata, M., S. Epstein and M. Baptista. 2015. Can crosslinguistically variant grammars be formally identical? Third factor underspecification and the possible elimination of parameters of UG. Lingua 156. 1-16.

Ott, D. and V. Struckmeier. 2018. Particles and deletion. Linguistic Inquiry 49: 393407.

Reis, M. 1992. The category of invariant alles in wh-clauses: On syntactic quantifiers vs. quantifying particles in German. In: Tracy, R. (ed.), Who climbs the grammar tree? Tübingen: Max Niemeyer Verlag. 465-492.

Rochman, L. 2005. The role of intonation in floating quantifiers. In: Blaho, S., L. Vicente and E. Schoorlemmer (eds.), Proceedings of ConSOLE XIII. 313-330.

Selkirk, E. 1995. Sentence prosody: Intonation, stress and phrasing. In: Goldsmith, J.A. (ed.), The handbook of phonological theory. Oxford: Blackwell. 550-569.

Shlonsky, U. 1991. Quantifiers as functional heads: a study of quantifier float in Hebrew. Lingua 84. 159-180.

Sportiche, D. 1988. A theory of floating quantifiers and its corollaries for constituent structure. Linguistic Inquiry 19. 425-449.

Takahashi, D. 1994. Minimality of Movement. (PhD dissertation, University of Connecticut, Storrs.)

Urban, E. 1999. Exactly stranding. (Ms., University of California, Santa Cruz.)

Wiland, B. 2010. Overt evidence from left-branch extraction in Polish for punctuated paths. Linguistic Inquiry 41. 335-347. 
Corresponding author:

Andreas Blümel

Seminar für Deutsche Philologie

University of Göttingen

Jacob-Grimm-Haus

Käte-Hamburger-Weg 3

D-37073 Göttingen

ablueme@gwdg.de 